\title{
Inverted-T pattern reduction mammoplasty in bilateral breast ptosis: cosmetic and oncological outcomes
}

\author{
Huayu Hu ${ }^{1,2}$, Qingyu Guan ${ }^{1,2}$, Yiqiong Zheng ${ }^{2}$, Yuting Zhong ${ }^{2,3}$, Ningning Min ${ }^{1,2}$, Yufan Wei ${ }^{1,2}$, \\ Rui Geng ${ }^{2,3}$, Chenyan Hong ${ }^{1,2}, \mathrm{Jie} \mathrm{Li}^{2}$, Yanjun Zhang ${ }^{2}$, Xiru $\mathrm{Li}^{2}$ \\ ${ }^{1}$ School of Medicine, Nankai University, Tianjin, China; ${ }^{2}$ Department of General Surgery, Chinese People's Liberation Army General Hospital, \\ Beijing, China; ${ }^{3}$ Medical School of Chinese PLA, Beijing, China \\ Contributions: (I) Conception and design: X Li, Y Zhang; (II) Administrative support: X Li; (III) Provision of study materials or patients: X Li; (IV) \\ Collection and assembly of data: H Hu, Q Guan; (V) Data analysis and interpretation: All authors; (VI) Manuscript writing: All authors; (VII) Final \\ approval of manuscript: All authors. \\ Correspondence to: Xiru Li; Yanjun Zhang. Department of General Surgery, Chinese People's Liberation Army General Hospital, Beijing 100853, \\ China. Email: 24681i@sina.com; zhangyanjun301@163.com.
}

Background: Breast ptosis is directly caused by Cooper's ligament laxity, with the decline of nipple areola complex (NAC) and mammary parenchyma. Breast cancer with ptosis is always a knotty problem that can hardly be repaired by classic breast conservation surgery (BCS) ending up with a pleasing appearance. We analyzed our 12 years' experience of performing inverted-T pattern techniques to treat bilateral breast ptosis, with or without breast cancer.

Methods: One hundred forty-eight breasts in 74 patients undergoing inverted-T pattern reduction mammoplasty were included in this study. Information about patients' clinical and surgical characteristics, complications, NAC sensitivity, cosmetic and oncological outcomes were collected and retrospectively analyzed.

Results: In the cohort of 57 patients with pure breast ptosis, the mean body mass index (BMI) was $25.2 \mathrm{~kg} / \mathrm{m}^{2}$, and the mean weight of resected tissue from the left and right breast reductions were 744.9 and $756.7 \mathrm{~g}$. In the cohort of 17 patients diagnosed as breast cancer with ptosis, the mean BMI was $25.1 \mathrm{~kg} / \mathrm{m}^{2}$, and the mean weight of resected tissue were $504.1 \mathrm{~g}$ for left and $535.6 \mathrm{~g}$ for right side. The majority of repairs were performed for tumors located in the upper outer $(58.8 \%)$, mostly with inferior or superomedial pedicles (90\%). All the upper inner tumors were repaired with inferior pedicles. Minor complications such as seroma $(8.1 \%)$, NAC epidermolysis $(8.1 \%)$, delayed wound healing $(4.1 \%)$ were detected postoperatively. Partial NAC necrosis occurred in one patient (1.4\%). $82.4 \%$ of all the patients rated "very satisfied" or "satisfied" as the final cosmetic outcomes. NAC sensitivity was "very high" and "high" in $82.4 \%$ patients. No local occurrence, distant metastasis and mortality occurred in tumor patients.

Conclusions: The inverted-T pattern reduction mammoplasty is a reliable technique to treat bilateral breast ptosis with a low complication rate. For cases with breast cancer, this technique can achieve both satisfying cosmetic outcomes and oncological safety.

Keywords: Breast ptosis; breast cancer; invert-T pattern; reduction mammoplasty

Submitted Aug 17, 2021. Accepted for publication Sep 17, 2021.

doi: 10.21037 /gs-21-561

View this article at: https://dx.doi.org/10.21037/gs-21-561 


\section{Introduction}

The breast maintains its normal position by Cooper's ligaments which anchor the glandular lobe to the superficial fascia of the skin and pectoralis major fascia. Breast ptosis is directly caused by ligamentous laxity, with the decline of nipple areola complex (NAC) and mammary parenchyma. Additionally, women with elder age, significant weight loss, multiple pregnancies and history of smoking were found vulnerable to suffer breast ptosis (1). Breast ptosis is not only aesthetically unpleasing but also brings physical and mental problems like submammary eczema, cervicodynia, omalgia and reduced self-esteem. According to Regnault's review in 1976 (2), true breast ptosis is classified into different grades based on the position relationship between NAC and inframammary fold (IMF): "grade I" for NAC located at the projection height of IMF and above the gland bottom; "grade II" for NAC below the projection height of IMF and above the gland bottom; "grade III" for NAC located at the lowest point of breast contour. Nowadays, different surgical strategies are devised to treat breast ptosis, in which the inverted-T pattern reduction mammoplasty is one of the most popular and time-tested techniques, especially for high-grade breast ptosis or gigantomastia that need to be resected amounts of skins.

Breast cancer with ptosis is always a knotty problem for surgeons. Previously, mastectomy was routinely applied to resect the unilateral ptotic breast with tumor tissue, but the significant weight difference of both sides could cause tough walk and scoliosis postoperatively. So, it is necessary to perform bilateral operations to ensure the burden equilibrium of both sides. Although breast conservation surgery (BCS) followed by radiotherapy has been proved equivalent with mastectomy in overall or disease-free survival and become another appropriate choice (3), classic BCS techniques can hardly guarantee an acceptable cosmetic result for patients with ptotic type of breast cancer. Oncoplastic surgery (OPS) serves as a rising approach evolved from BCS and ensures a massive tissue removal and better symmetry. Clough et al. (4) summarized an Atlas and classification for OPS, among which the inverted-T technique was performed for resections ranging $20-50 \%$ of the breast volume (level II OPS). Currently the inverted-T pattern techniques matched with suitable pedicles have been adopted for oncoplastic mammoplasty and received satisfying outcomes $(5,6)$. However, more evidences are needed to further demonstrate the reliability of this technique.
In this study, we retrospectively analyzed our 12 years' experience of performing inverted-T pattern techniques to treat bilateral breast ptosis, with or without breast cancer, aiming to provide more technical details as well as cosmetic and oncological outcomes. We present the following article in accordance with the STROBE reporting checklist (available at https://dx.doi.org/10.21037/gs-21-561).

\section{Methods}

\section{Patients}

The study was conducted in accordance with the Declaration of Helsinki (as revised in 2013). The study was approved by Medical Ethics Committee of Chinese PLA General Hospital (No. S2021-491-01) and informed consent was taken from all the patients. From January 2009 to August 2020, 91 patients diagnosed as breast ptosis in our department were selected. Seventeen patients were excluded because of the loss to follow-up and inadequate clinical data. The remaining 74 patients undergoing reduction mammoplasty (148 breasts) were included and analyzed in this study, among which 17 patients were simultaneously detected cancer in ptotic breast and other 57 patients were diagnosed as pure breast ptosis. All of them were female Chinese patients and received inverted-T pattern reduction mammoplasty (level II OPS for breast cancer patients) (4).

\section{Data collection}

The preoperative data contained age, height, weight, body mass index (BMI), menstrual status, physical examination, mammary ultrasound (all ages) and mammography (age $>40$ ). The resected tissue was weighed during the operation. The parenchymal pedicles applied in breast surgery were recorded. The appearance photography of breast before and after operation was documented for comparison.

Oncological data included size, location, histological characteristics of tumor, as well as lymph node staging and incisal margins. Magnetic resonance imaging (MRI) was obtained to exclude suspected multicentricity in cancer patients.

\section{Surgical techniques}

Initially, the Wise keyhole inverted-T pattern markings were made in all the patients (Figure 1A). All markings 
A
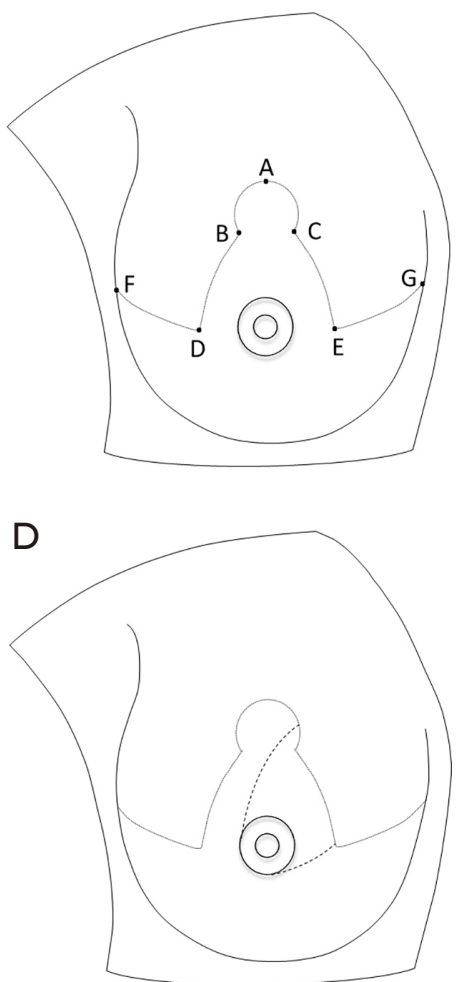

B

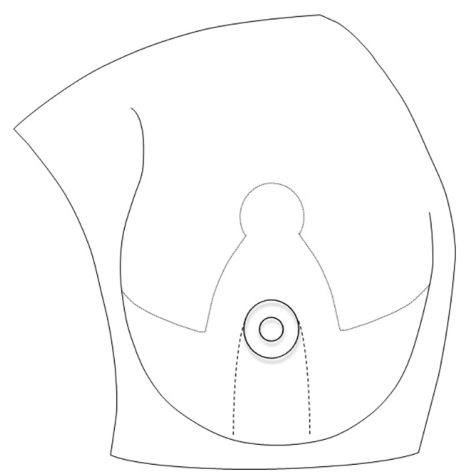

$\mathrm{E}$

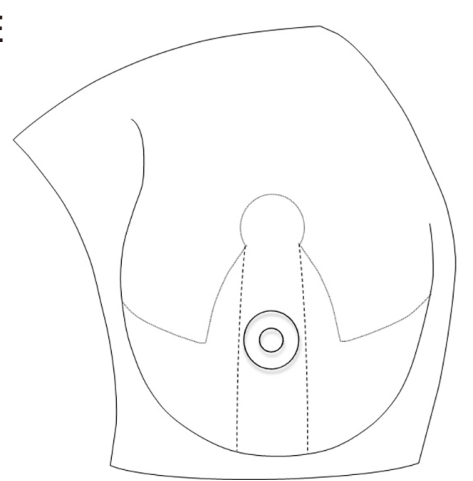

C

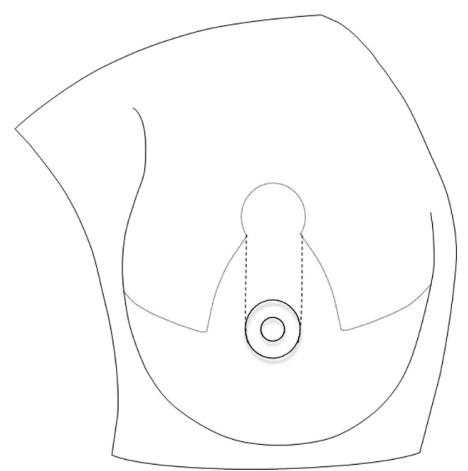

Figure 1 Surgical technique and markings. (A) Preoperative skin markings depict a Wise pattern. Details of drawing are described in Methods. (B-E) Design of inferior, superior, superomedial and vertical bipedicle.

and measurements were finished preoperatively when patients stood upright. The new nipple was positioned at the projection of IMF over the clavicular midline (point A), about $18-20 \mathrm{~cm}$ away from middle clavicle point and 9-11 cm away from midsternal line. Then draw a circle (about 4-5 cm diameter) where the new NAC locates, with point $\mathrm{A}$ as the vertex, point $\mathrm{B}$ at the 8 o'clock position and point $\mathrm{C}$ at the 4 o'clock position. Extend point $\mathrm{B}$ by $6-8 \mathrm{~cm}$ to $\mathrm{D}$ and point $\mathrm{C}$ to $\mathrm{E}$ symmetrically. Line $\mathrm{BD}$ or $\mathrm{CE}$ represented the distance from new NAC to IMF and forms the vertical scar of "inverted T". Then we drew an arc from point $\mathrm{E}$ to parasternal line (intersected at point $\mathrm{G}$ ), and similarly did markings from point $\mathrm{D}$ to anterior axillary line (intersected at point F). The IMF line formed the base margin of the pattern. Subsequently, an inferior, superior, superomedial pedicle or vertical bipedicle (Figure 1B-1E) was marked, ensuring that the tumor was not directly below the pedicle and was included in the resection area. In order to achieve symmetry, the same pedicle was adopted on the contralateral breast.
Once general anesthesia was completed, the pedicle was deepithelialized after injected into $0.9 \%$ saline with epinephrine (1:250,000) (Figure 2). The pedicle was incised with a scalpel down to pectoralis fascia and undermined at $2-\mathrm{cm}$ thickness at the nipple. Then a submammary tunnel was undermined to prepare for the accommodation of the dermoglandular flap. The tumor and surplus breast tissue were resected intactly and weighted for approximate removal of both sides. The incisal margin of tumor tissue must be proved negative pathologically, otherwise a second removal should be made to reach the oncological safety standard. A meticulous coagulation was made to protect the vascularity and innervation of NAC pedicle. The breast tissue with pedicle was pulled or rotated to fill out the submammary tunnel, and fixed to the pectoralis major fascia using 2-0 nylon sutures to reshape the breast cone (7). At the same time the NAC was brought to its new position. There is no amputation design with a free nipple graft in all operations. Negative pressure drainage was put in both sides of breast. Then the remaining abutments were sutured 

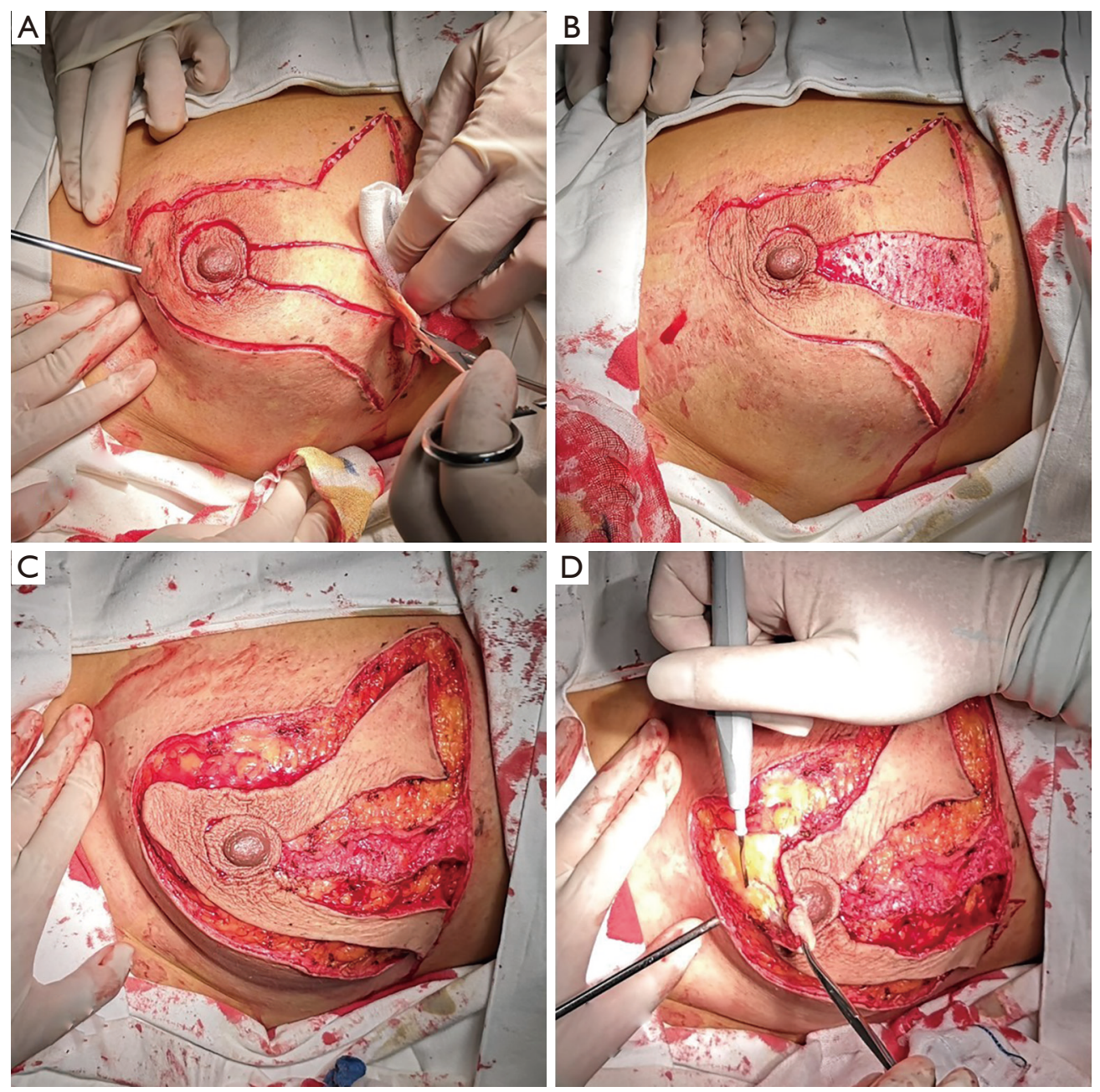

Figure 2 Deepithelialization according to preoperative skin markings and dissection of surrounding skin (A-D). The inferior pedicle was incised with a scalpel down to pectoralis major fascia. The excess breast tissue and subcutaneous fat was resected en bloc with the superior pole.

and an inverted-T scar formed. Skin stapler was sometimes used in cases with a tight stretching force to reinforce the suture.

\section{Pre- and postoperative treatment}

It's necessary for patients with breast cancer to receive pre- and postoperative comprehensive therapy. Inverted-T pattern OPS is essentially a technique developing from BCS, so all patients accepted radiotherapy postoperatively (50 Gy on the breast and additional 16 Gy on the tumor bed plus draining lymphatics when encroached). Adjuvant chemotherapy and endocrine therapy were considered depending on pathological results according to national comprehensive cancer network (NCCN) guidelines.

\section{Follow-up}

Patients with pure breast ptosis were asked to the outpatient clinic for incision dressing change and drainage nursing every 3 days for the first 3 weeks and thereafter at 4 months for final examination. Patients carrying ptotic breast with cancer were followed up routinely at 6 months to 1 -year intervals in case of tumor recurrence or distant metastasis. Complications and medical photography were documented during follow-up for surgery evaluation. Telephone survey 
Table 1 Clinical and surgical characteristics of patients

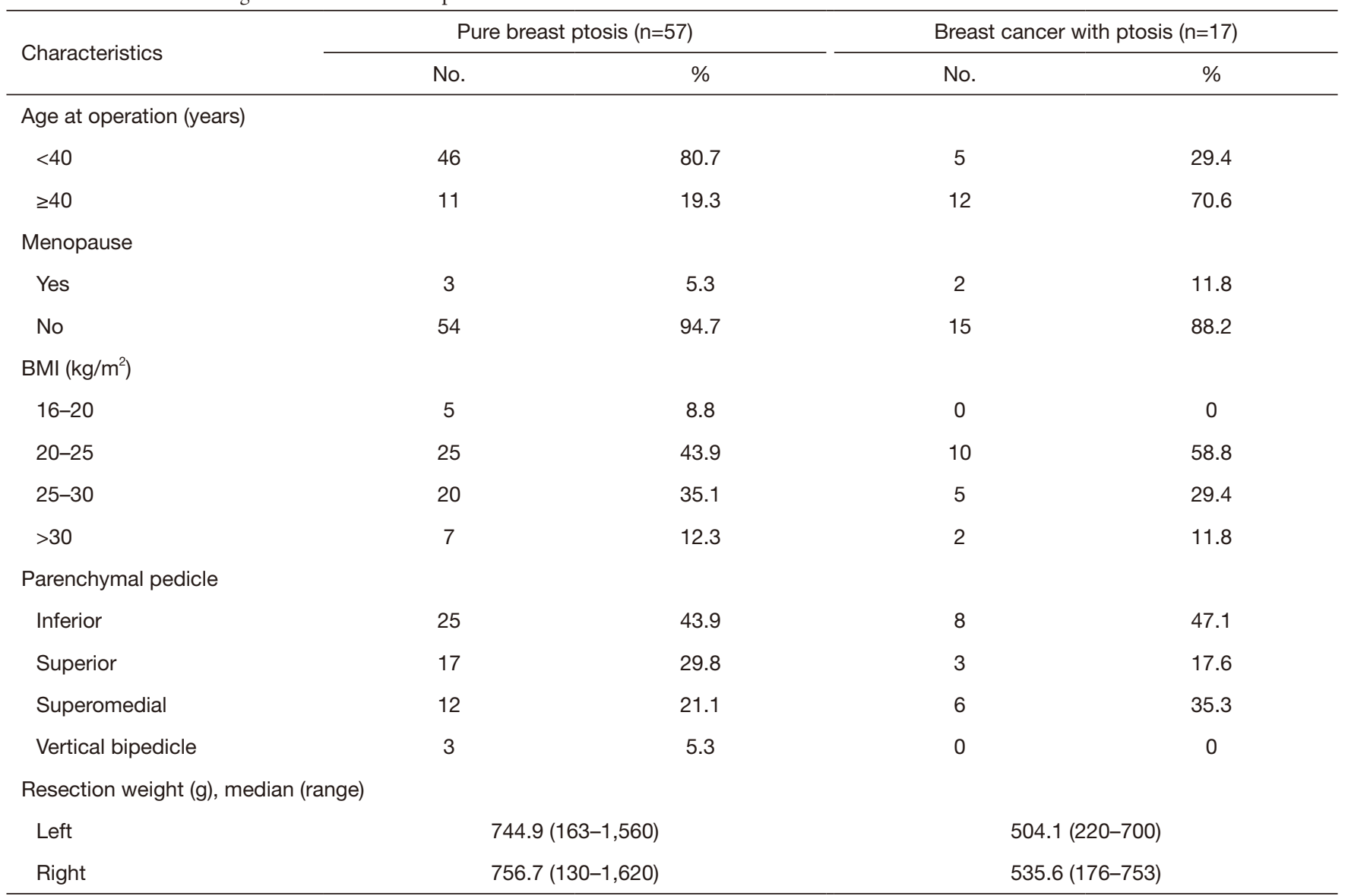

BMI, body mass index.

was also applied when necessary.

\section{Outcome evaluation}

Structured questionnaires were sent to patients undergoing inverted-T pattern surgery 1 year latter to collect patient's opinion about the outcome: (I) satisfaction about the aesthetic results in several aspects (scars, symmetrization, breast fullness and contour deformity) on a 5 -point scale ( $1=$ very satisfied, $2=$ satisfied, $3=$ moderate, $4=$ less satisfied, $5=$ unsatisfied); and (II) sensitivity of NAC one year after the procedure using Semmes-Weinstein method (1= very high, $2=$ high, $3=$ medium, $4=$ low, $5=$ no sensation). Subsequently, the feedbacks were summarized and analyzed by surgeons.

\section{Statistical analysis}

All data statistics were analyzed by SPSS 23.0, standard version (SPSS Inc., Chicago, IL, USA). Results were described as means with associated median and range. The $\chi^{2}$ test and Fisher exact test were used for comparison of categorical variables. $\mathrm{P}$ value $<0.05$ was considered significant statistical difference.

\section{Results}

The clinical and surgical characteristics of patients were shown in Table 1. All the patients received bilateral breast reduction to obtain appearance symmetry. In the cohort of pure breast ptosis, the average age of the patients was 34.8 years (range, $19-55$ years). The mean BMI was $25.2 \mathrm{~kg} / \mathrm{m}^{2}$ (range, $17.6-34.0 \mathrm{~kg} / \mathrm{m}^{2}$ ). Fifty-four patients $(94.7 \%)$ were in premenopausal state. Sternal notch-tonipple distance varied from 19 to $28 \mathrm{~cm}$, with a mean of $22.3 \mathrm{~cm}$. Operative time varied from 120 to $201 \mathrm{~min}$, with a mean of $139 \mathrm{~min}$. The mean weight of resected 
Table 2 Association between breast tumor quadrant and pedicle design

\begin{tabular}{lccc}
\hline & \multicolumn{3}{c}{ Pedicle design } \\
\cline { 2 - 4 } Tumor quadrant & $\begin{array}{c}\text { Inferior } \\
(\mathrm{n}=8)\end{array}$ & $\begin{array}{c}\text { Superior } \\
(\mathrm{n}=3)\end{array}$ & $\begin{array}{c}\text { Superomedial } \\
(\mathrm{n}=6)\end{array}$ \\
\hline Upper outer $(\mathrm{n}=10)$ & 4 & 1 & 5 \\
Upper inner $(\mathrm{n}=2)$ & 2 & 0 & 0 \\
Lower outer $(\mathrm{n}=3)$ & 1 & 1 & 1 \\
Lower inner $(\mathrm{n}=2)$ & 1 & 1 & 0 \\
\hline
\end{tabular}

tissue from the left and the right breast reductions were 744.9 and $756.7 \mathrm{~g}$, respectively.

In the cohort of breast cancer with ptosis, the average age was 35.1 years (range, 31-63 years) and the mean BMI was $25.1 \mathrm{~kg} / \mathrm{m}^{2}$ (range, $20.3-34.1 \mathrm{~kg} / \mathrm{m}^{2}$ ). About 88.2 percent of patients were premenopausal. Sternal notch-to-nipple distance varied from 20 to $26 \mathrm{~cm}$, with a mean of $21.5 \mathrm{~cm}$. Operative time varied from 150 to $221 \mathrm{~min}$, with a mean of $165 \mathrm{~min}$. The mean removal of breast tissue was $504.1 \mathrm{~g}$ (range, 220-700 g) for the left side and $535.6 \mathrm{~g}$ (range, 176$753 \mathrm{~g}$ ) for the right side. The primary tumors were classified as follows: Tis (ductal carcinoma in situ), one patient (5.9\%); T1, eleven patients $(64.7 \%)$; T2, five patients (29.4\% \%). Lymph nodes status was classified as follows: N0, thirteen patients (76.5\%); N1, two patients (11.8\%); $\mathrm{N} 2$, two patients (11.8\%). All tumors obtained a negative incision margin for the primary resection according to intraoperative pathological results. Only one patient $(5.9 \%)$ received neoadjuvant chemotherapy and thirteen (76.5\%) patients completed postoperative chemotherapy. Fourteen $(82.4 \%)$ patients received endocrine treatment and all the seventeen patients finished standard radiotherapy described in methods.

Table 1 lists various designs of the parenchymal pedicles used in all patients. $73.7 \%$ of patients in the cohort of pure breast ptosis were performed operations with superior or inferior parenchymal pedicles; while in patients with breast cancer, inferior or superomedial pedicles were preferred, accounting for $82.4 \%$ in total. Table 2 shows the association between breast tumor quadrant and pedicle design. Most repairs were performed for tumors located in the upper outer $(58.8 \%)$. Breasts invaded by upper outer tumors were mostly repaired with inferior or superomedial pedicles (90\%); all the upper inner quadrant tumors were repaired with inferior pedicles.
Most complications in this study are minor and controllable. In all 74 patients, seroma (8.1\%), NAC epidermolysis $(8.1 \%)$, delayed wound healing $(4.1 \%)$ were detected during incision checking. Unfortunately, a partial NAC necrosis occurred in the case with a superior pedicle and a secondary revision was conducted (1.4\%). No pedicle necrosis was seen in our study.

Patients were interviewed during the follow-up in outpatient clinic or by telephone. The follow-up duration was 12-151 months (mean, 90 months). The cosmetic outcomes and NAC sensitivity evaluation from patients were listed in Table 3, revealing a high satisfaction ratio. In the cohort of pure breast ptosis, 25 patients (43.9\%) rated the results as "very satisfied" and 22 patients (38.6\%) as "satisfied"; NAC sensitivity was rated by patients in 21 NACs (36.8\%) as "very high" and in 27 NACs (47.4\%) as "high". In the cohort of breast cancer with ptosis, 8 patients $(47.1 \%)$ rated the results as "very satisfied" and 6 patients $(35.3 \%)$ as "satisfied"; NAC sensitivity was rated by patients in 6 NACs (35.3\%) as "very high" and in 7 NACs (41.2\%) as "high". On the whole, $82.4 \%$ of all the patients were "very satisfied" or "satisfied" with the final cosmetic outcomes and NAC sensitivity was "very high" and "high" in $82.4 \%$ patients. Two typical cases were shown and the pre-and postoperative appearances were illustrated in Figures 3,4.

During the follow-up, no local occurrence, distant metastasis and mortality was observed in all 17 patients diagnosed as breast cancer with ptosis.

\section{Discussion}

Over the last decades, the surgical techniques for breast reduction were constantly innovating but still challenging in problems such as unacceptable contour, ptosis recurrence and unsatisfied scars. Various mammoplasty types including periareolar, vertical, inverted-T pattern were reported for breast reshaping (8). The periareolar or round block technique was suitable for mild and moderate breast ptosis with a limited tissue removal (9). When the excess skin did not fit the remaining gland, the redundancy of the outer round could gather and lead to pleating. Vertical scar technique popularized by Lejour was proud for the short vertical scar and rapid operative times, compared with inverted-T, but tended to create an exaggerated upper pole and sloped inferior pole (8). Inverted-T or Wise pattern mammoplasty was a well-established technique and applied in a wide range of breast sizes. Despite relatively burdensome scars, this technique was familiar in breast 
Table 3 Results of cosmetic satisfaction and NAC sensitivity in inverted T techniques

\begin{tabular}{|c|c|c|c|c|c|c|}
\hline Outcome parameters & \multicolumn{2}{|c|}{ Pure breast ptosis $(n=57)$} & \multicolumn{2}{|c|}{ Breast cancer with ptosis $(n=17)$} & \multicolumn{2}{|c|}{ Total $(n=74)$} \\
\hline \multicolumn{7}{|l|}{ Cosmetic satisfaction } \\
\hline $1=$ very satisfied & 25 & 43.9 & 8 & 47.1 & 33 & 44.6 \\
\hline $2=$ satisfied & 22 & 38.6 & 6 & 35.3 & 28 & 37.8 \\
\hline $4=$ less satisfied & 4 & 7.0 & 2 & 11.8 & 6 & 8.1 \\
\hline $5=$ unsatisfied & 2 & 3.5 & 0 & 0 & 2 & 2.7 \\
\hline \multicolumn{7}{|l|}{ NAC sensitivity } \\
\hline $1=$ very high & 21 & 36.8 & 6 & 35.3 & 27 & 36.5 \\
\hline $4=$ low & 5 & 8.8 & 2 & 11.8 & 7 & 9.5 \\
\hline $5=$ no sensation & 1 & 1.8 & 0 & 0 & 1 & 1.4 \\
\hline
\end{tabular}

NAC, nipple areolar complex.
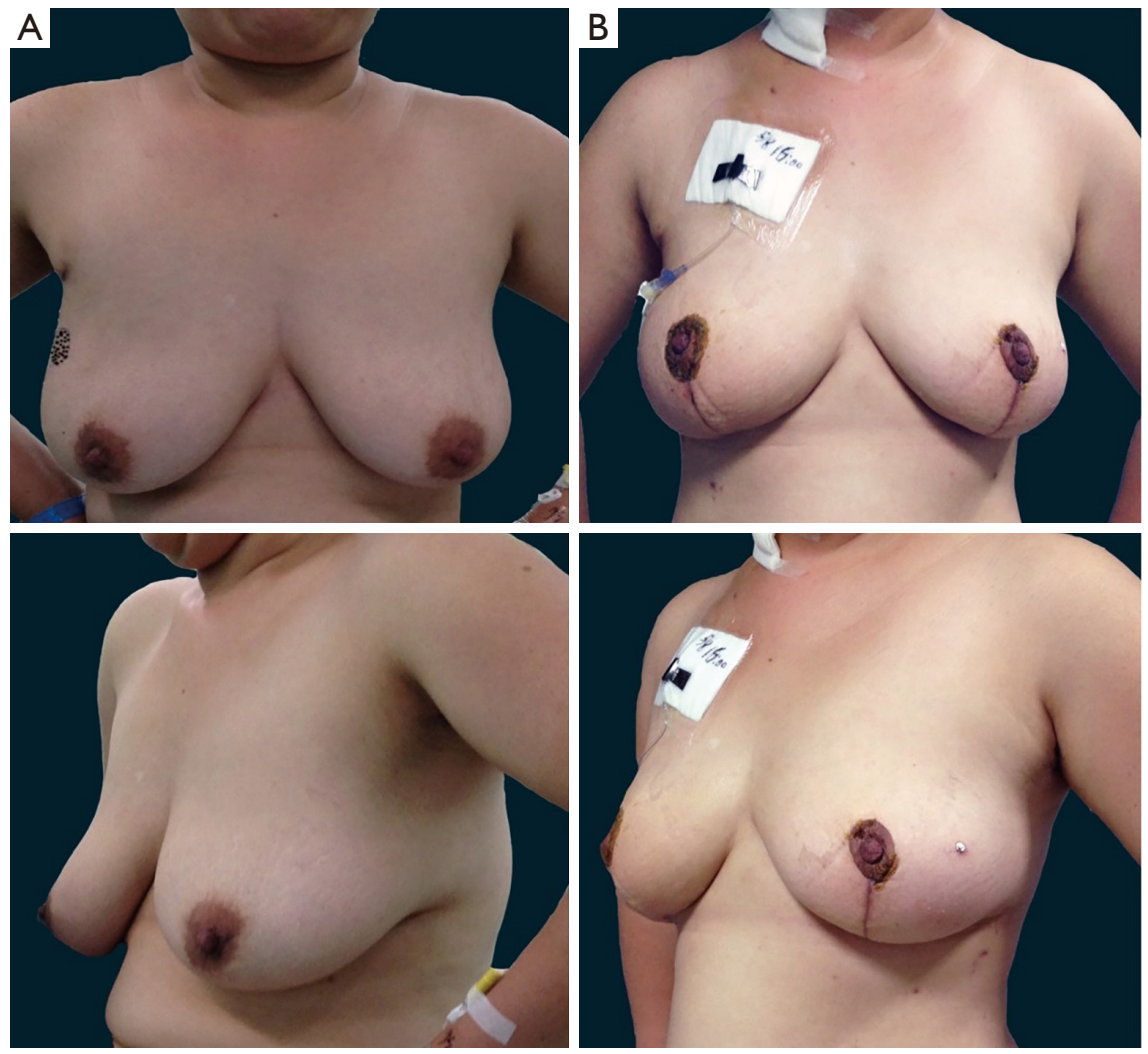

Figure 3 A 32-year-old woman with an invasive ductal carcinoma located in upper outer quadrant as well as a grade I (Regnault method) breast ptosis. An inverted-T pattern breast reduction using an inferior pedicle was performed. Preoperative (A) and 4 weeks postoperative photos (B) were illustrated. The patient rated "very satisfied" for the appearance. This image is published with the patient's consent. 

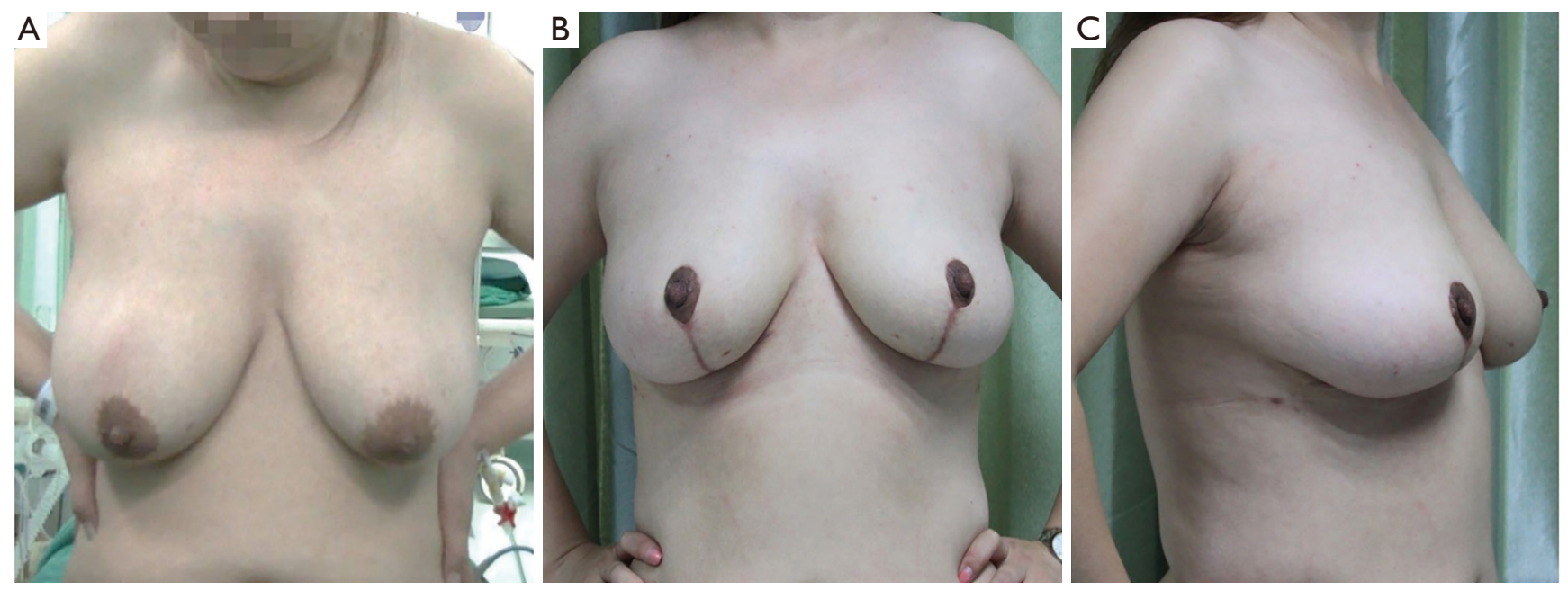

Figure 4 A 33-year-old woman, with pure ptosis grade II by Regnault, received a superomedial-pedicle reduction mammoplasty. Preoperative (A) and 4 weeks postoperative photos $(B, C)$ were shown. The patient was "very satisfied" with the breast shape. This image is published with the patient's consent.

and plastic surgeons with the widest spread in the world. With the advancement of OPS, inverted-T technique was adopted as a breast conservation approach for breast cancer treatment using volume replacement theory, particularly for women with severely ptotic breast $(4,6,10)$.

The relocation of NAC plays a crucial role in breast reduction. Free nipple grafting has been outdated for the areola depigmentation and sensitivity loss while pedicled breast reduction yielded successful results in many cases $(11,12)$. Actually, we think the understanding of mammary vascular territories can help preserve the vitality of pedicles or NAC, and free nipple grafting is seldom used in our department. The superficial blood supply comes mainly from the internal mammary artery, the thoracic and thoracoacromial system (13). The four pedicles reported in our study, superior, inferior, superomedial pedicle and vertical bipedicle, are therefore rational anatomical options. Pitanguy introduced the superior pedicle in the 1960s (14). Mckissock described vertical bipedicle in 1972 (15). Robbins published the idea with inferior pedicle in 1977 and gained popularity (16). Orlando described the superomedial pedicle in 1975 (17). All these pedicle techniques were demonstrated to be reliable and safe in various literatures $(6,11,18,19)$.

Our study indicated that breast tumor location had an influence on pedicle design (Table 2). Tumors located in upper outer quadrant tended to be reconstructed with inferior or superomedial pedicles, while tumors in upper inner quadrant were all repaired with inferior pedicles. Besides, most quadrants of tumors could be repaired with inferior pedicles, while superomedial pedicles were mainly performed in tumors positioned in upper outer quadrant. The frequency of superior pedicle was the least in all three types. The reason of such distribution, we thought, is that superior pedicles were usually fold up and superomedial pedicles with the whole base were rotated into appropriate position when the NAC was lifting to a new position (13). By comparison, the inferior pedicle maintained straight and was pulled upwards together with NAC, providing a favorable fullness of inferior pole. Additionally, the widespread of inferior technique enabled surgeons to have a preferable master of this design.

Complications were relatively rare in our study. The negative pressure drainage effectively decreased the incidence of seroma $(8.1 \%)$. NAC epidermolysis was detected in 8.1 percent of patients and all of them healed without obvious scars. Nipple necrosis occurred in one case with superior pedicle and we supposed it is ascribed to the narrow deepithelialized flap, reminding us that we cannot ignore the thickness of superior pedicle, or enlarge its width. Due to the incorporation of skin stapler, no "T junction" breakdown was seen in the whole cohort. Delayed wound healing was also uncommon (4.1\%).

Questionnaires collected from patients indicated a high level of satisfaction about the cosmetic outcomes. Despite the large resected volume, all the cases achieved an excellent symmetry 
and almost inconspicuous malformation (Figures 3,4). Our experience is trying to ensure that the same pedicle is performed on contralateral side. The "inverted-T" scars were inevitable in most cases. Hence, for inverted-T mammoplasty, it is advisable to strictly choose patients those who are anticipated to have major asymmetry using traditional BCS technique. NAC sensitivity and viability is an important indicator of a successful pedicle design. In this study, the majority of cases maintained a high sensitivity of NAC and one case had an unsensible NAC. We suggest that a careful dissection is indispensable when operating near the pedicle with a surgical electrotome, and the high-frequency coagulation function should be used as little as possible.

Oncological safety is always a concern for OPS procedure. Owing to a larger resection of breast tissue, the positive incision margin rates were much lower in OPS techniques than that in classic BCS process (10-12\% vs. 19-40\%) (20). Clough et al. (21) reported a $1.7 \%$ of local recurrence rate using oncoplastic techniques based on quadrant-perquadrant atlas (4). Sanchez et al. (6) confirmed the excellent oncological results of level II OPS spanning 20 years, with an overall survival of $93.7 \%$ and a disease-free survival of $82.3 \%$. Our study further demonstrated a good prognosis for this technique, with no tumor-related recurrence and mortality for a median of 90 months' follow-up.

Some limitations exist in this retrospective study. The overall number of patients in the cohort of breast cancer with ptosis is small. Besides, there is no preoperative survey on the sensitivity of NAC for a comparison. The aesthetic evaluation is based on a five-point scale system measured by patients and more objective parameters may be needed. Detailed information and data will be supplemented in our further studies.

In conclusion, the inverted-T pattern incision for reduction mammoplasty is a reliable technique to treat bilateral breast ptosis with a low complication rate. For cases with breast cancer, this technique can achieve both satisfying cosmetic outcomes and oncological safety.

\section{Acknowledgments}

Funding: None.

\section{Footnote}

Reporting Checklist: The authors have completed the STROBE reporting checklist. Available at https://dx.doi. org/10.21037/gs-21-561
Data Sharing Statement: Available at https://dx.doi. org/10.21037/gs-21-561

Peer Review File: Available at https://dx.doi.org/10.21037/ gs-21-561

Conflicts of Interest: All authors have completed the ICMJE uniform disclosure form (available at https://dx.doi. org/10.21037/gs-21-561). XL serves as an Editor-in-Chief of Gland Surgery from May 2017 to April 2022. YZ serves as an unpaid Associate Editor of Gland Surgery from March 2018 to February 2023. The other authors have no conflicts of interest to declare.

Ethical Statement: The authors are accountable for all aspects of the work in ensuring that questions related to the accuracy or integrity of any part of the work are appropriately investigated and resolved. The study was conducted in accordance with the Declaration of Helsinki (as revised in 2013). The study was approved by Medical Ethics Committee of Chinese PLA General Hospital (No. S2021-491-01) and informed consent was taken from all the patients.

Open Access Statement: This is an Open Access article distributed in accordance with the Creative Commons Attribution-NonCommercial-NoDerivs 4.0 International License (CC BY-NC-ND 4.0), which permits the noncommercial replication and distribution of the article with the strict proviso that no changes or edits are made and the original work is properly cited (including links to both the formal publication through the relevant DOI and the license). See: https://creativecommons.org/licenses/by-nc-nd/4.0/.

\section{References}

1. Rinker B, Veneracion M, Walsh CP. Breast ptosis: causes and cure. Ann Plast Surg 2010;64:579-84.

2. Regnault P. Breast ptosis. Definition and treatment. Clin Plast Surg 1976;3:193-203.

3. Fisher B, Anderson S, Bryant J, et al. Twenty-year followup of a randomized trial comparing total mastectomy, lumpectomy, and lumpectomy plus irradiation for the treatment of invasive breast cancer. N Engl J Med 2002;347:1233-41.

4. Clough KB, Kaufman GJ, Nos C, et al. Improving breast cancer surgery: a classification and quadrant per quadrant atlas for oncoplastic surgery. Ann Surg Oncol 
2010;17:1375-91.

5. Resende Paulinelli R, de Oliveira VM, Bagnoli F, et al. Oncoplastic mammaplasty with geometric compensation: Evolution of the technique, outcomes and followup in a multicentre retrospective cohort. J Surg Oncol 2020;121:967-74.

6. Sanchez AM, Franceschini G, D'Archi S, et al. Results obtained with level II oncoplastic surgery spanning 20 years of breast cancer treatment: Do we really need further demonstration of reliability? Breast J 2020;26:125-32.

7. Uebel CO, Piccinini PS, Ramos RFM, et al. Breast Reduction: The Superolateral Dermoglandular Pedicle Revisited. Aesthetic Plast Surg 2019;43:36-45.

8. Qureshi AA, Myckatyn TM, Tenenbaum MM. Mastopexy and Mastopexy-Augmentation. Aesthet Surg J 2018;38:374-84.

9. Liu Y, Ren Y, Wu M, et al. Simultaneous Mastopexy via Areola Excision to Correct Mild and Moderate Breast Ptosis. Aesthetic Plast Surg 2021;45:948-55.

10. Gunn J, Dortch J, TerKonda S, et al. Comparing morbidity rates between wise pattern and standard horizontal elliptical mastectomy incisions in patients undergoing immediate breast reconstruction. Breast J 2019;25:20-5.

11. Bustos SS, Molinar V, Kuruoglu D, et al. Inferior pedicle breast reduction and long nipple-to-inframammary fold distance: How long is safe? J Plast Reconstr Aesthet Surg 2021;74:495-503.

12. Basaran K, Saydam FA, Ersin I, et al. The free-nipple breast-reduction technique performed with transfer of the nipple-areola complex over the superior or superomedial pedicles. Aesthetic Plast Surg 2014;38:718-26.

Cite this article as: $\mathrm{Hu} \mathrm{H}$, Guan Q, Zheng Y, Zhong Y, Min N, Wei Y, Geng R, Hong C, Li J, Zhang Y, Li X. Inverted-T pattern reduction mammoplasty in bilateral breast ptosis: cosmetic and oncological outcomes. Gland Surg 2021;10(10):29252934. doi: $10.21037 / g s-21-561$
13. Hall-Findlay EJ, Shestak KC. Breast Reduction. Plast Reconstr Surg 2015;136:531e-44e.

14. Pitanguy I. Surgical treatment of breast hypertrophy. Br J Plast Surg 1967;20:78-85.

15. McKissock PK. Reduction mammaplasty with a vertical dermal flap. Plast Reconstr Surg 1972;49:245-52.

16. Robbins TH. A reduction mammaplasty with the areolanipple based on an inferior dermal pedicle. Plast Reconstr Surg 1977;59:64-7.

17. Orlando JC, Guthrie RH Jr. The superomedial dermal pedicle for nipple transposition. Br J Plast Surg $1975 ; 28: 42-5$.

18. Kronowitz SJ, Hunt KK, Kuerer HM, et al. Practical guidelines for repair of partial mastectomy defects using the breast reduction technique in patients undergoing breast conservation therapy. Plast Reconstr Surg 2007;120:1755-68.

19. Wolter A, Fertsch S, Munder B, et al. Double-Unit Superomedio-Central (DUS) Pedicle Inverted-T Reduction Mammaplasty in Gigantomastia: A 7-year Single-Center Retrospective Study. Aesthetic Plast Surg 2021. [Epub ahead of print]. doi: 10.1007/s00266-02102351-y.

20. Chatterjee A, Dayicioglu D, Khakpour N, et al. Oncoplastic Surgery: Keeping It Simple With 5 Essential Volume Displacement Techniques for Breast Conservation in a Patient With Moderate- to Large-Sized Breasts. Cancer Control 2017;24:1073274817729043.

21. Clough KB, Ihrai T, Oden S, et al. Oncoplastic surgery for breast cancer based on tumour location and a quadrantper-quadrant atlas. Br J Surg 2012;99:1389-95. 